\title{
Menus de substitution dans les cantines scolaires : un cocktail laïque?
}

Anne Fornerod

\section{Q OpenEdition}

12 Journals

Édition électronique

URL : https://journals.openedition.org/rdr/1648

DOI : $10.4000 /$ rdr. 1648

ISSN : 2534-7462

Éditeur

Presses universitaires de Strasbourg

\section{Édition imprimée}

Date de publication : 2 décembre 2021

Pagination : 177-183

ISBN : 979-10-344-0097-3

ISSN : 2493-8637

\section{Référence électronique}

Anne Fornerod, "Menus de substitution dans les cantines scolaires : un cocktail laïque ? ", Revue du droit des religions [En ligne], 12 | 2021, mis en ligne le 02 décembre 2021, consulté le 03 mai 2022. URL : http://journals.openedition.org/rdr/1648; DOI : https://doi.org/10.4000/rdr.1648

\section{(c) $(1)(9$}

La revue du droit des religions est mise à disposition selon les termes de la Creative Commons Attribution - Pas d'Utilisation Commerciale 4.0 International - CC BY-NC 4.0. 


\title{
Menus de substitution dans les cantines scolaires : un cocktail laïque?
}

\author{
Anne FORNEROD \\ Université de Strasbourg / CNRS, Droit, Religion, Entreprise et Société (DRES)
}

I 'annonce du maire de Chalon-sur-Saône en mars 2015 de ne plus proL poser de menus de substitution lorsqu'un plat contenant du porc serait servi dans les cantines scolaires de la ville ainsi que la délibération du conseil municipal actant cette décision à la rentrée suivante ont donné lieu à une suite juridictionnelle en plusieurs temps. Des deux jugements ${ }^{1}$ en première instance à l'arrêt du Conseil d'État du 11 décembre 2020 (n 426483), cette pluralité est marquée par la diversité des motivations des juridictions administratives. Elles illustrent le statut particulier des cantines scolaires, comme service public, mais également comme «annexe» de l'institution scolaire par leur vocation à prolonger l'apprentissage ${ }^{2}$ et la difficulté à échapper au questionnement sur les modalités d'application du principe de laïcité dans cette enceinte.

1. Dans une ordonnance du 12 août 2015, le tribunal administratif de Dijon avait rejeté la requête de la Ligue de défense judiciaire des musulmans, estimant que l'accès à la cantine des enfants de confession musulmane n'était pas compromis (TA Dijon, 12 août 2015, $\mathrm{n}^{\circ}$ 1502101, Ligue de défense judiciaire des musulmans). V. aussi TA Dijon, 28 août 2017 , $\mathrm{n}^{\circ} 1502100,1502726$, Ligue de défense judiciaire des musulmans et $a$.: A. FORNEROD, « Menus de substitution dans les cantines scolaires: de la laïcité à l'intérêt supérieur de l'enfant», Revue du droit des religions, $\mathrm{n}^{\circ}$ 5, 2018, p. 181-188.

2. V. les conclusions du rapporteur public Laurent Cytermann, qui s'ouvrent sur cette remarque: la cantine scolaire «est un lieu d'apprentissage, sans doute pas toujours de la gastronomie mais sûrement de la vie sociale. C'est à l'aune de ces enjeux qu'il faut appréhender l'affaire dont vous êtes saisis». 
La prise en considération par un service public d'une pratique religieuse consistant en une interdiction de consommer de la viande de porc (pratique qui réunit les religions juive et musulmane) soulevait la question de la place à lui accorder, de la singularité des usagers que sont les élèves, et des textes à mobiliser. Après l'originalité de la solution du tribunal administratif de Dijon et un certain classicisme en appel (1), le Conseil d'État opte pour une formule hybride dans laquelle deux visions du principe de laïcité se côtoient (2).

\section{DE L'INTÉRÊT DE L'ENFANT AU BON FONCTIONNEMENT D'UN SERVICE PUBLIC FACULTATIF}

Le tribunal administratif de Dijon avait offert une solution originale en examinant l'affaire à l'aune de l'intérêt supérieur des enfants. Il avait en effet fondé l'annulation de la décision de l'édile chalonnais sur ce qu'elle «affecte de manière suffisamment directe et certaine la situation des enfants fréquentant une cantine scolaire et constitue ainsi une décision dans l'appréciation de laquelle son auteur doit, en vertu de l'article 3-1 de la CIDE, accorder une attention primordiale à l'intérêt supérieur de l'enfant».

Le principe de laïcité n'est pas convoqué, si ce n'est pour désavouer le conseil municipal qui avait adopté une "position de principe se référant à une conception de la laïcité» pour justifier de mettre fin à la pratique des menus de substitution. En revanche, même après avoir rappelé le caractère facultatif de la restauration scolaire et constaté l'absence de texte prévoyant une obligation pour la mairie de proposer aux enfants un menu de substitution $^{3}$, le tribunal estime que le choix offert à travers un tel menu "permettait la prise en compte, dans le respect de la liberté de conscience des enfants et des parents, de préoccupations d'ordre religieux ou culturel ».

3. Contrairement, par exemple, à ce qui vaut pour les personnes détenues, l'article 9 du règlement-type des établissements pénitentiaires annexé à l'article R. 57-6-18 du Code de procédure pénale prévoyant que «chaque personne détenue reçoit une alimentation variée, bien préparée et présentée, répondant tant en ce qui concerne la qualité que la quantité aux règles de la diététique et de l'hygiène, compte tenu de son âge, de son état de santé, de la nature de son travail et, dans toute la mesure du possible, de ses convictions philosophiques ou religieuses ». Le Conseil d'État en avait pourtant déduit qu'« en vertu de ces dispositions, il appartient à l'administration pénitentiaire, qui n'est pas tenue de garantir aux personnes détenues, en toute circonstance, une alimentation respectant leurs convictions religieuses, de permettre, dans toute la mesure du possible eu égard aux contraintes matérielles propres à la gestion de ces établissements et dans le respect de l'objectif d'intérêt général du maintien du bon ordre des établissements pénitentiaires, l'observance des prescriptions alimentaires résultant des croyances et pratiques religieuses» (CE, 10 févr. 2016, n 285929). 
Dans son argumentation, la cour administrative d'appel de Lyon $^{4}$ avait quant à elle mis l'accent strictement sur le cadre juridique d'un service public facultatif. Selon elle, si ce caractère facultatif offre à l'administration gestionnaire «de larges pouvoirs d'organisation», elle «ne peut toutefois décider d'en modifier les modalités d'organisation et de fonctionnement que pour des motifs en rapport avec les nécessités de ce service». De là, elle estime que «les principes de laïcité et de neutralité auxquels est soumis le service public» ne peuvent faire partie de ces motifs et qu' «en l'absence de nécessité se rapportant à son organisation ou son fonctionnement, les usagers du service public facultatif de la restauration scolaire se voient offrir un choix leur permettant de bénéficier d'un menu équilibré sans avoir à consommer des aliments proscrits par leurs convictions religieuses ou philosophiques». En outre, à l'instar du tribunal administratif de Dijon - et avant que ne le fasse également le Conseil d'État - la cour rappelle que la pratique consistant à proposer un menu de substitution existait dans les restaurants scolaires des écoles publiques de la ville depuis 1984, écartant ainsi l'argument de difficultés financières ou techniques du service.

Son raisonnement, focalisé sur le fonctionnement du service, revenait d'une certaine façon à limiter l'incidence du caractère facultatif du service de restauration scolaire, allant dans le sens du nouvel encadrement législatif adopté l'année précédente - et d'un certain pragmatisme, dans la mesure où la restauration scolaire «existe dans $80 \%$ des communes dotées d'une école publique $^{5} »$ - puisque, depuis la loi no 2017-86 du 27 janvier 2017, l'article L. 131-13 du Code de l'éducation dispose que «l'inscription à la cantine des écoles primaires, lorsque ce service existe, est un droit pour tous les enfants scolarisés. Il ne peut être établi aucune discrimination selon leur situation ou celle de leur famille ${ }^{6} »$. Ces dispositions sont issues d'un amendement procédant du constat que «dans les années récentes, plusieurs communes ont refusé le droit d'accès aux cantines scolaires aux élèves dont au moins l'un des parents n'exerçait pas d'activité professionnelle ${ }^{7} »$, sachant que «le plus souvent, ce refus d'accès concerne des élèves dont l'un des parents au

4. CAA Lyon, 23 oct. 2018, n 17LY03323.

5. Conclusions du rapporteur public, p. 1.

6. V. Rapport fait au nom de la commission spéciale, chargée d'examiner, après engagement de la procédure accélérée, le projet de loi «égalité et citoyenneté, Ass. nationale, 17 juin 2016, $\mathrm{n}^{\mathrm{O}} 3851$, p. 138, ainsi que, sur l'interprétation de ces dispositions: L. ERSTEIN, «(Presque) tous à la cantine ou la relativité du droit à...», JCP A 2021, act. 204.

7. Amendement $\mathrm{n}^{\circ} 46$ présenté par M. Schwartzenberg, Mme Hobert et M. Carpentier, Ass. nationale, 7 juin 2016 . 
moins est au chômage. Ce qui revient à discriminer, voire à stigmatiser des familles déjà en difficulté». Selon les auteurs de cet amendement, "on ne peut admettre une discrimination, fondée sur la situation des enfants et celle de leur famille, qui séparerait les élèves les uns des autres au moment des repas et qui mettrait à l'écart les plus défavorisés».

Ce faisant, la cour examinait la question de la prise en compte des prescriptions religieuses alimentaires à la lumière de la logique classique de contrainte du bon fonctionnement d'un service public, laissant le principe de laiicité à la marge. Le Conseil d'État, en revanche, emprunte un chemin pour ainsi dire opposé, remettant le principe de laïcité au cour de sa décision.

\section{DE LA LAÏCITÉ DU SERVICE PUBLIC À LA LAÏCITÉ DE L'ÉCOLE PUBLIQUE ?}

Le Conseil d'État retient une solution allant dans le même sens que les juridictions inférieures dans cette affaire en jugeant qu'«il n'existe aucune obligation pour les collectivités territoriales gestionnaires d'un service public de restauration scolaire de distribuer à ses usagers des repas différenciés leur permettant de ne pas consommer des aliments proscrits par leurs convictions religieuses», mais que "ni les principes de laïcité et de neutralité du service public, ni le principe d'égalité des usagers devant le service public, ne font, par eux-mêmes, obstacle à ce que ces mêmes collectivités territoriales puissent proposer de tels repas». En outre, «lorsque les collectivités ayant fait le choix d'assurer le service public de restauration scolaire définissent ou redéfinissent les règles d'organisation de ce service public, il leur appartient de prendre en compte l'intérêt général qui s'attache à ce que tous les enfants puissent bénéficier de ce service public, au regard des exigences du bon fonctionnement du service et des moyens humains et financiers dont disposent ces collectivités». Le Conseil d'État opère une synthèse entre les deux décisions précédentes, à deux nuances significatives près.

D'une part, l'intérêt supérieur de l'enfant cède la place à l'intérêt général dont on peut supposer qu'il consiste à «accueillir à une même table républicaine l'ensemble des scolarisés ${ }^{8}{ }$. D'autre part et surtout, il resitue clairement l'affaire sur le terrain de la laïcité. Certes, à la suite du tribunal administratif et de la cour administrative d'appel, il censure la démarche du maire de Chalon-sur-Saône consistant à instrumentaliser le principe de laïcité à des

8. M. Touzeil-Divina, «Laïcité des menus dits de substitution: la République n’oblige pas au porc», JCP A 2020, act. 737. 
fins opposées à ce que ce principe permet en matière de liberté religieuse, autrement dit à retenir une conception de la laicité comme synonyme d'une neutralité qui commanderait à l'autorité publique d'ignorer les convictions religieuses des usagers afin de ne pas établir de distinction fondée sur ces convictions. Le Conseil d'État n'en propose pas moins une lecture qui laisse quelque peu perplexe. Il s'écarte du raisonnement de la cour d'appel en jugeant que «c'est par un motif surabondant que la cour a énoncé que le gestionnaire d'un service public facultatif ne peut décider d'en modifier les modalités d'organisation et de fonctionnement que pour des motifs en rapport avec les nécessités du service, dès lors qu'elle jugeait que la commune, qui n'avait fondé les décisions litigieuses que sur l'invocation des principes de laïcité et de neutralité du service public, ne pouvait pas légalement se fonder sur ces seuls principes pour décider de ne plus servir de menus de substitution». Or, si «c'est bien à la conception même de la laïcité que le Conseil d'État devait apporter une réponse ${ }^{9} »$, il livre une solution quelque peu hybride.

Il convoque en effet deux visions du principe de laïcité présentes dans la jurisprudence du Conseil constitutionnel. Ce sont pourtant des philosophies différentes qui sous-tendent les deux définitions données à près de dix ans d'intervalle. La seconde, chronologiquement, correspond à celle de la décision QPC du 21 février 2013 qui conjugue les dispositions de l'article $1^{\text {er }}$ de la Constitution de 1958 et les articles 1 et 2 de la loi du 9 décembre 1905 pour affirmer que la laïcité recouvre aussi bien la neutralité de l'État que la liberté religieuse dans ses deux dimensions, interne et externe (cons. 5). Reprenant mot pour mot le considérant constitutionnel, le Conseil d'État s'abstient toutefois de faire une quelconque référence à la liberté religieuse des usagers du service public - dont relève la possibilité d'observer des interdits alimentaires découlant de prescriptions religieuses - même s'il est entendu que son exercice doit se plier aux exigences du service. La Charte de la laïcité dans les services publics, en 2007, reconnaît que les usagers «ont le droit d'exprimer leurs convictions religieuses dans les limites du respect de la neutralité du service public, de son bon fonctionnement et des impératifs d'ordre public, de sécurité, de santé et d'hygiène». La Haute juridiction aurait pu, à la suite de la cour d'appel, se contenter d'affirmer qu'en présence d'un service public facultatif, les collectivités territoriales ne sont pas tenues de prendre en compte les convictions religieuses des usagers sur le plan alimentaire et de leur servir des repas en conséquence.

9. H. Pauliat, « Pas d'interdiction des menus de substitution dans les cantines scolaires au nom de la laïcité», JCP A 2021, 2026. 
Pour autant, le Conseil d'État poursuit en citant cette fois la décision du Conseil constitutionnel du 19 novembre 2004 qui définit le principe de laïcité par l'interdiction «à quiconque de se prévaloir de ses croyances religieuses pour s'affranchir des règles communes régissant les relations entre collectivités publiques et particuliers ${ }^{10} »$. Cette inspiration révèle l'incidence ici du cadre scolaire sur la problématique des menus de substitution et la force centripète qu'y exerce la laïcité. Les enfants semblent davantage considérés comme des élèves que comme des usagers d'un service public (facultatif). Il n'est sans doute pas dû au hasard que «la question de la distinction concrète de l'agent et de l'usager » se soit "posée à propos des parents d'élèves apportant leur concours au service public scolaire, soit pour encadrer des sorties scolaires ${ }^{11} »$ et alors que, jusqu'à la loi du 15 mars 2004, le juge administratif avait aménagé pour l'expression des convictions religieuses des élèves (qu'il s'agisse du port de signes religieux ou d'autorisations d'absence) un «régime relativement libéral ${ }^{12}$ ». Or, il a été fait observer que dans la définition de la laïcité de la décision du 19 novembre 2004 le Conseil constitutionnel «met indirectement l'accent sur les principes de neutralité et d'égalité» et «valide implicitement la législation particulière existant en France, à savoir la loi du 15 mars 2004 encadrant, en application du principe de laïcité, le port de signes ou de tenues manifestant une appartenance religieuse dans les écoles, collèges et lycées publics ${ }^{13}{ }$. Cette référence du Conseil d'État en 2020 surprend au regard de la réception juridique, sociale et politique de ce texte, qui symbolise l'introduction dans une loi de la «laïcité axiologique ${ }^{14}$ ». Il convient pour mieux comprendre de se tourner vers les conclusions du rapporteur public qui, tout en affirmant que la loi du 15 mars 2004 «procède de l'idée que le comportement des élèves eux-mêmes peut porter atteinte à la laïcité», semble défendre la pertinence et la pérennité de la jurisprudence libérale antérieure et assure que cette loi «n'a nullement remis en cause cette faculté de prise en compte des confessions religieuses dans le fonctionnement du service public». Si la lisibilité de la solution retenue in fine en est affectée, elle demeure par ailleurs équilibrée et appropriée à la situation

10. Cons. const., 19 nov. 2004, n 2004-505 DC, Traité établissant une Constitution pour l'Europe.

11. G. Eveillard, «La licéité des menus de substitution dans les cantines scolaires», Dr. adm. 2021, comm. 16.

12. Ibid.

13. L. BAKIR, «Laïcité et institution scolaire: variations dans l'application d'un principe juridique», Revue du droit des religions, $\mathrm{n}^{\circ}$ 2, 2016, p. 123-138.

14. L. BAKIR, «Réflexions autour de la laïcité axiologique», Revue du droit des religions, $\mathrm{n}^{\circ} 8$, 2019, p. 137-154. 
«dans une période où la crainte des séparatismes et des communautarismes est patente ${ }^{15} »$.

Ainsi, même si la question des menus végétariens pourrait détrôner celle des menus de substitution dans la médiatisation des cantines scolaires ${ }^{16}$, il semble que la laïcité appliquée aux élèves ait encore des enseignements à livrer.

15. H. Pauliat, art. cit.

16. Indépendamment de la polémique soulevée par la décision de la municipalité écologiste de Lyon de proposer des menus sans viande dans les cantines scolaires au printemps 2021, deux questions parlementaires illustrent cette tendance. Alors qu'en septembre 2019, un député faisait observer qu'«incidemment, cette alternative [végétarienne] permettrait de régler définitivement l'instrumentalisation politicienne de la question des menus scolaires. Les plats végétariens permettraient en respectant la laïcité, de permettre à tous les enfants d'avoir une alimentation suffisamment protéinée lors des repas scolaires, indépendamment de leurs éventuels interdits alimentaires liés à une religion» (Rép. min. no 1302: JOAN, 31 oct. 2017, p. 5290), trois ans plus tard, la question du menu végétarien est clairement déliée de celle des menus de substitution pour n'être rattachée qu'à son fondement législatif, à savoir l'article L. 230-5-6 du Code rural et de la pêche maritime, introduit par l'article 24 de la loi pour l'équilibre des relations commerciales dans le secteur agricole et alimentaire et une alimentation saine, durable et accessible à tous, loi dite EGALIM. Depuis le $1^{\text {er }}$ novembre 2019, chaque établissement de restauration scolaire (de la maternelle au lycée) doit proposer, une fois par semaine, un menu diversifié végétarien (Rép. min. $\mathrm{n}^{\circ}$ 32284: JOAN, 5 janv. 2021, p. 62). 